\title{
AVALIAÇÃO DO PERFIL DE LIBERAÇÃO DO FÁRMACO IBUPROFENO EM MEMBRANAS SIMÉTRICAS E ASSIMÉTRICAS DE ACETATO DE CELULOSE: EFEITO DA MORFOLOGIA
}

\author{
Marcos V. Ferreira ${ }^{\mathrm{a}}$, Lauro A. Pradela Filho ${ }^{\mathrm{a}}$, André L. dos Santos ${ }^{\mathrm{a}, \mathrm{b}}$, Regina M. Takeuchi ${ }^{\mathrm{a}, \mathrm{b}}$ e Rosana M. N. de Assunção ${ }^{\mathrm{a}, \mathrm{b}, *, \mathbb{B}}$ \\ anstituto de Química, Universidade Federal de Uberlândia, campus Santa Mônica, 38400-902 Uberlândia - MG, Brasil \\ 'Instituto de Ciências Exatas e Naturais do Pontal, Universidade Federal de Uberlândia, campus Pontal, 38304-402 Ituiutaba - \\ MG, Brasil
}

Recebido em 21/04/2019; aceito em 30/07/2019; publicado na web em 11/09/2019

\begin{abstract}
EVALUATION OF THE IBUPROFEN DELYVERY PROFILE IN SYMMETRIC AND ASYMMETRIC MEMBRANES OF CELLULOSE ACETATE: EFFECT OF MORPHOLOGY. Cellulose derivatives still receive special attention in today biomedical applications due to their biocompatibility, ability to form resistant membranes and compatibility with various bioactive agents. In this work, cellulose acetate (CA) was used to prepare membranes with different morphologies aiming to evaluate the release profile of the drug ibuprofen (IBF). The IBF incorporation was performed by the addition of $10 \%$ of the drug in an $8 \%$ (w/w) CA/acetone solution. The morphology of the membranes was modified by the addition of water, used as porogenic agent. Drug release assays showed the effect of morphology on the cumulative drug release curves. The symmetric membrane exhibited retention of significant amounts of the drug and a slow release process that lasted for more than 50 hours. The asymmetric porous membranes presented different behavior with greater amount of drug released in less time of release. The kinetic parameters calculated from the Higuchi and Korsmeyer/Peppas models indicated release profiles controlled by diffusion/solution process in the polymer matrix. The results revealed that the interactions established between IBF, CA and the matrix morphology are significant factors for the kinetic control of the drug release process and enable to choose different applications of the membranes loaded with IBF.
\end{abstract}

Keywords: cellulose acetate; membrane morphologies; ibuprofen; controlled release; Square-Wave Voltammetry.

\section{INTRODUÇÃO}

O desenvolvimento de sistemas para liberação controlada de fármacos a partir de novos polímeros ou a aplicação de polímeros já conhecidos em novas formulações tem recebido atenção de vários pesquisadores nas últimas décadas. ${ }^{1}$ Nesse sentido, derivados de celulose como acetato de celulose (AC) têm sido utilizados como matrizes poliméricas para liberação controlada devido à sua biocompatibilidade, capacidade de formação de membranas e micropartículas e sua elevada temperatura de transição vítrea. ${ }^{2,3}$ Esses sistemas de matrizes são frequentemente usados para prolongar, atrasar ou direcionar a taxa de liberação de fármacos, reduzindo o número de doses e a frequência das dosagens em comparação com aquelas formulações farmacêuticas que apresentam uma forma de liberação imediata (convencional) ${ }^{2,4}$ Yu et al. ${ }^{5}$ e Nista et al. ${ }^{6}$ por exemplo, empregaram membranas de AC como matrizes para liberação controlada de cetoprofeno e do sulfato de gentamicina, respectivamente, alcançando resultados satisfatórios no controle da liberação destes fármacos.

Sendo assim, o polímero AC se destaca ainda pela capacidade de formação de membranas com modificações morfológicas que podem ser controladas pela adição de um não-solvente (NS) comum e de baixo custo, como a água. Esse sistema trifásico AC-solvente-NS, quando empregado para formação de membranas pelo método de inversão de fases pela evaporação de solvente, caracteriza-se pela formação de uma estrutura porosa, cujos poros podem ter suas dimensões alteradas por variações nas proporções do NS e do solvente..$^{7-10}$ Essas modificações morfológicas influenciarão diretamente na forma como o fármaco será distribuído na matriz polimérica e nas interações estabelecidas entre a matriz polimérica e o fármaco, bem como na suscetibilidade que o sistema apresentará à entrada do solvente do meio de liberação. Consequentemente, a taxa de difusão do fármaco

*e-mail: rosana.assuncao@ufu.br poderá ser favorecida pelo aumento da quantidade e tamanho destes poros, fornecendo um mecanismo eficiente de controle para obtenção de uma determinada taxa de liberação do fármaco pretendida.

Dentre uma vasta gama de fármacos passíveis de estudos de liberação modificada, o ibuprofeno (fármaco anti-inflamatório não esteroidal, com efeitos analgésicos, antipiréticos e anti-inflamatórios) se notabiliza por ter aplicação tanto em sistemas que possuam um perfil de liberação rápida quando se objetiva efeitos antitérmicos e analgésicos, como em um perfil de liberação sustentada quando se deseja o tratamento contínuo da artrite reumatoide ou osteoartrite, por exemplo. ${ }^{11}$ IBF está disponível comercialmente, principalmente, na forma de comprimidos revestidos e suspensões em dosagens que variam entre 200 a $800 \mathrm{mg}$, administrados de três a quatro vezes por dia, respeitando um limite de $1200 \mathrm{mg} \mathrm{dia}^{-1}$ para administrações sem prescrição médica ou $2400 \mathrm{mg} \mathrm{dia}^{-1}$ sob prescrição médica, apresentando boa absorção oral. ${ }^{12}$ Suas concentrações séricas máximas são atingidas entre 1 a 2 horas após a administração oral, sendo rapidamente biotransformado com uma meia-vida no soro de 1,8 a 2 horas. ${ }^{13} \mathrm{O}$ fármaco é completamente eliminado em 24 horas após a última dose através do metabolismo. ${ }^{12,14}$

Posto isso, uma forma de avaliar e comparar novos sistemas matriciais desenvolvidos é pelo uso de equações genéricas que traduzem matematicamente a curva de dissolução do fármaco em função de alguns parâmetros relacionados às formas farmacêuticas, determinando parâmetros de liberação cinética de liberação in vitro do fármaco e contribuindo para o melhor entendimento das interações fármaco-polímero e o(s) mecanismo(s) que rege(m) a liberação dos fármacos. ${ }^{15}$

Neste trabalho, ressalta-se o uso da voltametria de onda quadrada (SWV, do inglês "Square-Wave Voltammetry") como técnica de deteccção e quantificação do fármaco, uma vez que, como observado por Fonseca et al., ${ }^{3}$ permite a realização de medidas em tempo real de forma rápida e eficiente, descartando etapas de preparação 
da amostra que poderiam culminar em perda do analito, além de apresentar limites de quantificação compatíveis às necessidades dos ensaios de liberação com fármacos quando comparada a outras técnicas analíticas, inclusive outras técnicas voltamétricas. ${ }^{3}$ Para tanto, destaca-se também os estudos realizados por e Švorc et al. ${ }^{16}$ que, além de descreverem o mecanismo de oxidação eletroquímica do ibuprofeno (IBF), conseguiram detectar e quantificar este fármaco através de medidas SWV empregando como eletrodo de trabalho um eletrodo de diamante dopado com boro.

Outras técnicas empregadas, como a Calorimetria Exploratória Diferencial (DSC, do inglês "Differential Scanning Calorimetry") e Microscopia Eletrônica de Varredura (MEV), também se constituem em ferramentas importantes para caracterizar e avaliar as matrizes poliméricas empregadas na liberação de fármacos, com a finalidade de obter informações importantes relacionadas à interação fármaco-polímero, morfologia da matriz e distribuição do fármaco em sua formulação. ${ }^{17-19}$ Dessa forma, neste trabalho foram produzidas membranas $\mathrm{AC}$ com diferentes morfologias para incorporação e liberação do IBF, empregado como fármaco modelo, avaliação de seus comportamentos sob ensaios de liberação de fármaco in vitro e aplicação de modelos cinéticos como o de Korsmeyer-Peppas, Weibull e Higuchi, a fim de determinar o mecanismo de transporte que rege a liberação do fármaco a partir destas membranas.

\section{PARTE EXPERIMENTAL}

Todos os reagentes utilizados neste estudo foram de grau de pureza analítica e puderam ser utilizados sem mais etapas de purificação.

\section{Produção das membranas simétricas e assimétricas de acetato de celulose (AC)}

Membranas simétricas foram preparadas pela dissolução do $\mathrm{AC}(8 \% \mathrm{~m} / \mathrm{m})$ em acetona (Dinâmica ${ }^{\circledR}$, Indaiatuba, Brasil) pelo método de evaporação de solvente adaptado. ${ }^{18} \mathrm{O} \mathrm{AC}$ foi fornecido gentilmente pela empresa Rhodia Solvay ${ }^{\circledR}$ (Santo André, Brasil), na forma de flocos e com grau de substituição (GS) igual a 2,45. Esse GS é definido como o número médio de grupos hidroxila que foram substituídos por grupos acetila, por unidade D-glicopiranosil da cadeia polimérica, podendo variar de 0 (para a celulose) a 3 (para o triacetato de celulose) e, assim, influenciar na solubilidade do polímero em um determinado solvente. Sendo assim, o AC fornecido pode ser classificado como um diacetato de celulose (GS entre 1,9 e 2,5) e, portanto, é sabidamente solúvel em acetona..$^{20,21} \mathrm{O}$ fármaco foi incorporado às membranas pela adição de $10 \%(\mathrm{~m} / \mathrm{m})$ de ibuprofeno/acetato de celulose (AC-SM/IBF) à solução de AC antes do espalhamento em placa de Petri. O padrão de IBF, $97 \%$ de pureza, foi adquirido em uma indústria de insumos farmacêuticos (Gemini LTDA, Anápolis, Brasil). Em seguida, a etapa de evaporação de solvente foi promovida a $25^{\circ} \mathrm{C}$ por $12 \mathrm{~h}$. Posteriormente, a membrana foi destacada e pesada. Procedimento semelhante foi adotado para a formação das membranas assimétricas (AC-AM/ $\mathrm{IBF})$, em que 10,15 e $25 \%(\mathrm{~m} / \mathrm{m})$ de água foram adicionadas, intentando-se sua ação como agente porogênico (não solvente para o sistema formado). Todas as membranas foram produzidas com dimensões de $9,5 \mathrm{~cm}$ de diâmetro.

\section{Caracterização morfológica e estrutural das membranas produzidas}

As membranas foram metalizadas com uma fina camada de ouro em uma metalizadora para carbono e ouro modelo Quorum QR 150ES e analisadas quanto à superfície e seção transversal das membranas em um Microscópio Eletrônico de Varredura modelo VEGA3, TESCAN, operando a 5 ou $10 \mathrm{kV}$.

As membranas também foram avaliadas por Calorimetria Exploratória Diferencial (DSC) em Calorímetro Exploratório modelo Q-20, da TA Instruments. Foram utilizados porta amostras padrão de alumínio com tampa, em que 4-5 mg da membrana foram aquecidos de 25 a $280{ }^{\circ} \mathrm{C}$ para registro de uma primeira varredura. Após a primeira varredura, o equipamento foi resfriado com gelo até que o forno atingisse a temperatura de $20{ }^{\circ} \mathrm{C}$ e submetido a novo aquecimento de 25 a $280{ }^{\circ} \mathrm{C}$ para registro de uma segunda varredura. As amostras foram analisadas na taxa de aquecimento de $10^{\circ} \mathrm{C} \mathrm{min}{ }^{-1} \mathrm{em}$ atmosfera de $\mathrm{N}_{2}$ em fluxo de $50 \mathrm{~cm}^{3} \mathrm{~min}^{-1}$.

\section{Ensaios de liberação in vitro do fármaco ibuprofeno (IBF)}

\section{Reagentes e Aparatos}

Soluções aquosas foram preparadas usando água ultrapura obtida através de um sistema de ultrapurificação Megapurity. Os experimentos voltamétricos foram realizados em um potenciostato/galvanostato $\mu$ AUTOLAB tipo III acoplado a um microcomputador e controlado por software NOVA 2.1. O pH das soluções foi medido com um pHmetro HANNA modelo HI 3221 acoplado a um eletrodo de vidro. Um agitador magnético com aquecimento da TECNAL modelo TE-0853 foi utilizado tanto para promover agitação quanto controlar a temperatura em $36{ }^{\circ} \mathrm{C}$ durante os ensaios de liberação do fármaco.

\section{Quantificação voltamétrica dos perfis de liberação do IBF}

Os perfis de liberação do IBF a partir das membranas foram estudados em um sistema de monitoramento por SWV, representado na Figura 1, e montado como segue: uma célula eletroquímica (A) contendo $10 \mathrm{~mL}$ de uma solução $0,1 \mathrm{~mol} \mathrm{~L}^{-1}$ de tampão fosfato, $\mathrm{pH} 7,4$, que atuou como eletrólito de suporte e fluido de simulação intestinal (FSI); membranas cortadas em pequenos pedaços e transferidas para a célula eletroquímica (B); medidas voltamétricas realizadas empregando-se um sistema de três eletrodos composto por um fio de Pt como eletrodo auxiliar (C), um eletrodo de $\mathrm{Ag} / \mathrm{AgCl} / \mathrm{KCl}_{\text {saturado }}$ $\left(\mathrm{AgCl}_{\text {sat }}\right)$ como eletrodo de referência (D) e um eletrodo de disco de carbono vítreo, com $3 \mathrm{~mm}$ de diâmetro, como eletrodo de trabalho (E); sistema mantido imerso em banho termostatizado a $36{ }^{\circ} \mathrm{C}(\mathrm{F})$, sob agitação magnética constante $(\mathrm{G})$, exceto durante as medidas voltamétricas. O IBF liberado pelas membranas foi quantificado por voltametria de onda quadrada (SWV), empregando-se os seguintes parâmetros voltamétricos: frequência de $150 \mathrm{~Hz}$, amplitude de pulso $75 \mathrm{mV}$ e incremento de varredura de $4 \mathrm{mV}$. Os voltamogramas foram registrados repetidamente em diferentes intervalos de tempo até que se atingisse uma porcentagem constante de fármaco liberado. Após cada medida voltamétrica, o eletrodo de carbono vítreo foi polido em uma suspensão de alumina com diâmetro de partícula de $5 \mu \mathrm{m}$ dispersa em um pano para polimento metalográfico.

\section{RESULTADOS E DISCUSSÃO}

\section{Caracterização morfológica e estrutural das membranas}

\section{Microscopia Eletrônica de Varredura (MEV)}

Membranas simétricas carregadas ou não com o fármaco IBF, foram produzidas na ausência do agente porogênico (água) e apresentaram aspecto transparente. As membranas assimétricas foram produzidas pela adição de diferentes proporções de água (10, 15 e $25 \% \mathrm{~m} / \mathrm{m}$ ), carregadas ou não com IBF, e apresentaram aspecto opaco. A opacidade da membrana pode ser um indício de irregularidades na distribuição de estruturas que causam a reflexão ou desvio da luz, podendo ser atribuídas a regiões cristalinas, poros (principal 


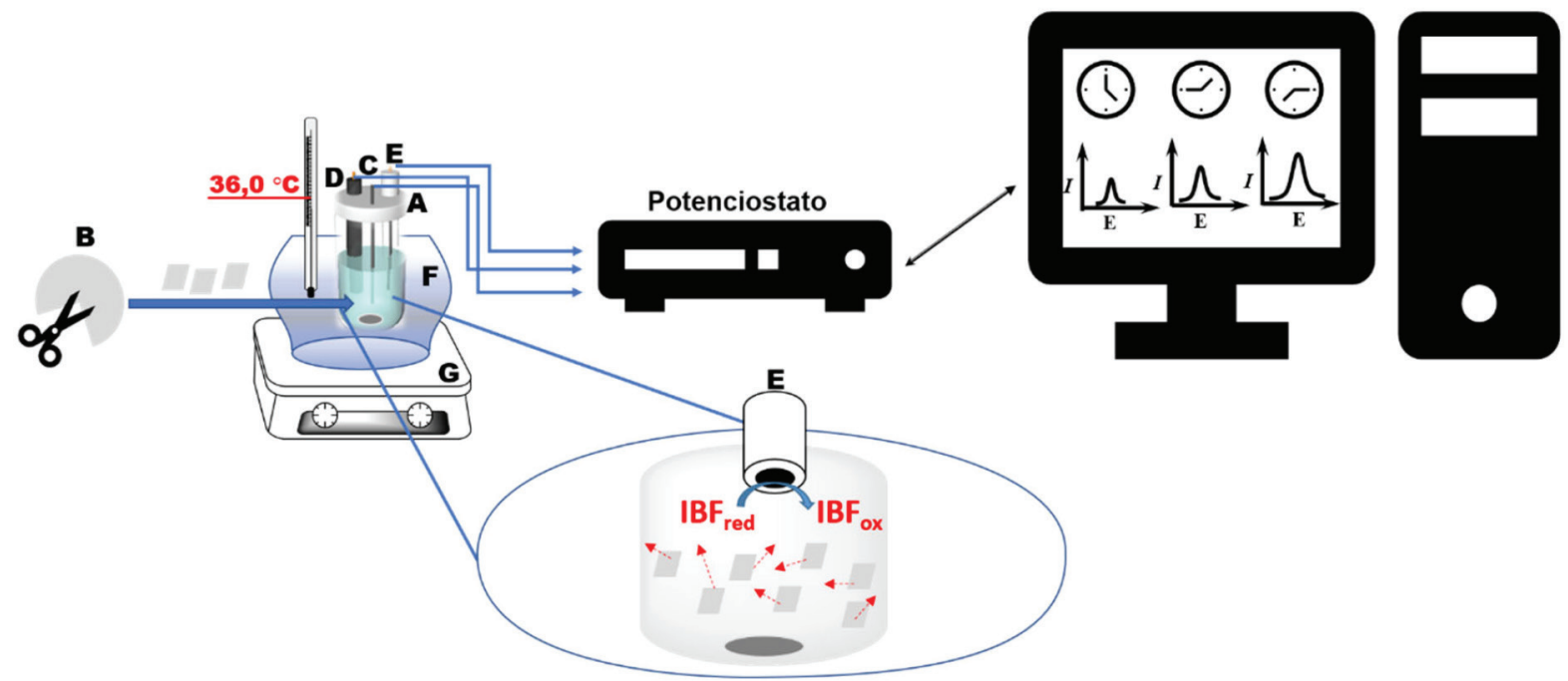

Figura 1. Fluxograma do sistema montado para monitoramento em tempo real do IBF liberado a partir das membranas

causa da opacidade nas membranas assimétricas) ou outras estruturas existentes nas membranas..$^{22}$ Imagens de duas membranas produzidas são mostradas na Figura 2.

As imagens por MEV das membranas referência de AC (sem fármaco) produzidas na ausência e na presença de diferentes proporções de água estão apresentadas na Figura 3. Fica evidente nessas imagens as modificações morfológicas produzidas pela presença do agente porogênico nas membranas.

Para AC-SM (Figura 3a) verifica-se que, na ausência de agente porogênico, a precipitação da membrana é condicionada somente pela evaporação do solvente e, assim, resulta na formação de uma membrana de superfície densa, contínua, homogênea e sem poros aparentes, na magnificação avaliada. Sua seção transversal (Figura 3b) evidencia a ausência de poros e exibe um aspecto de membrana formada por densas camadas estratificadas pela superposição das cadeias poliméricas, que justifica sua classificação como membrana do tipo simétrica densa.

As adições de diferentes proporções de agente porogênico (10, 15 e $25 \% \mathrm{~m} / \mathrm{m}$ - água/polímero) promoveram a formação de uma estrutura heterogênea com regiões mais densas associadas a precipitação da fase rica em polímero na presença de um não solvente (NS) e regiões mais porosas oriundas da precipitação da fase com menor concentração polimérica. Essa diferença de concentração polimérica é formada no interior da solução AC/acetona/água conforme transcorre a etapa de evaporação do solvente. Dessa forma, a proporção entre polímero/agente porogênico é responsável pelo aumento da quantidade, tamanho e formato dos poros. Com a adição de $10 \%(\mathrm{~m} / \mathrm{m})$ de água observa-se a formação de poros de diâmetros menores que $1 \mu \mathrm{m}$, interligados por regiões contínuas formadas pelo emaranhamento das cadeias poliméricas durante a fase de evaporação do solvente (Figura 3c). A imagem da seção transversal (na Figura 3d) exibe, ainda, poros com morfologia celular e comprimentos menores que $2,5 \mu \mathrm{m}$, sendo que alguns estão interconectados. Conforme pode ser visto nas Figuras 3e-h, o aumento da porcentagem de agente porogênico favorece a formação da fase polimérica menos concentrada, resultando em mudanças significativas na precipitação do polímero, evidenciadas pela formação de materiais pouco uniformes, com superfície e interior constituídos de poros, cujas dimensões também são proporcionais ao aumento da fração de agente porogênico. Essas morfologias descritas apresentam propriedades próximas das membranas classificadas como assimétricas e, assim, podem ser explicadas pelos processos de formação deste tipo de membrana pelo emprego do método de evaporação de solvente. ${ }^{10}$

A adição de qualquer substância à solução de preparação das membranas pode levar a mudanças morfológicas significativas devido à possibilidade de interação dessas espécies com o polímero. A Figura 4 apresenta as imagens por MEV para as membranas com IBF incorporado durante a produção das membranas.

Para as membranas preparadas com IBF sem agentes porogênicos, AC-SM/IBF (Figura 4a-b), a quantidade de fármaco adicionada não provocou mudanças significativas na morfologia da membrana que, semelhantemente à membrana de referência AC-SM (Figura 3a), apresenta-se como densa.
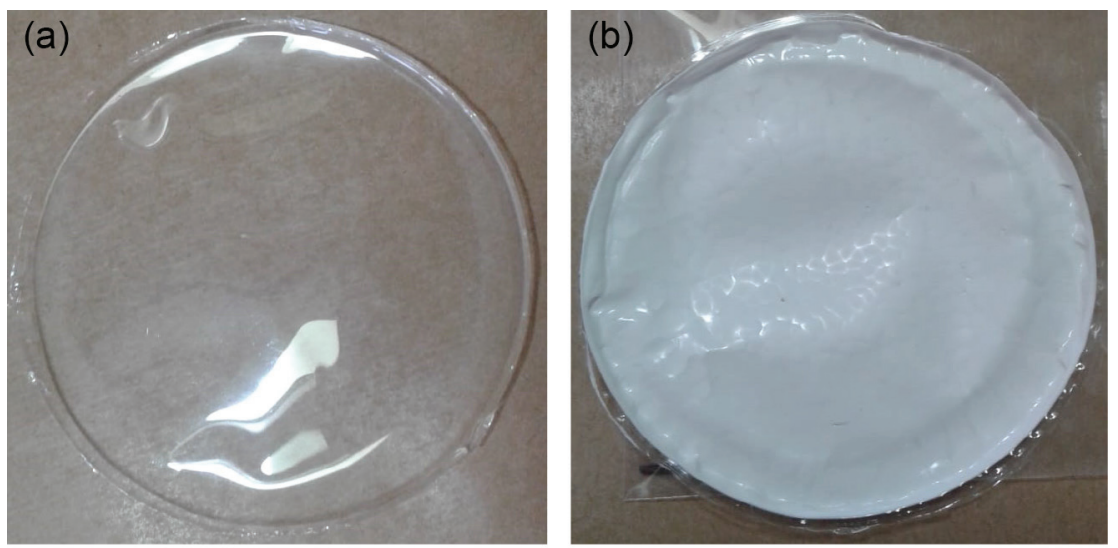

Figura 2. Fotografias (a) da membrana simétrica $(A C-S M)$ e (b) da membrana assimétrica com $15 \%$ de água (m/m) (AC-AM15) 

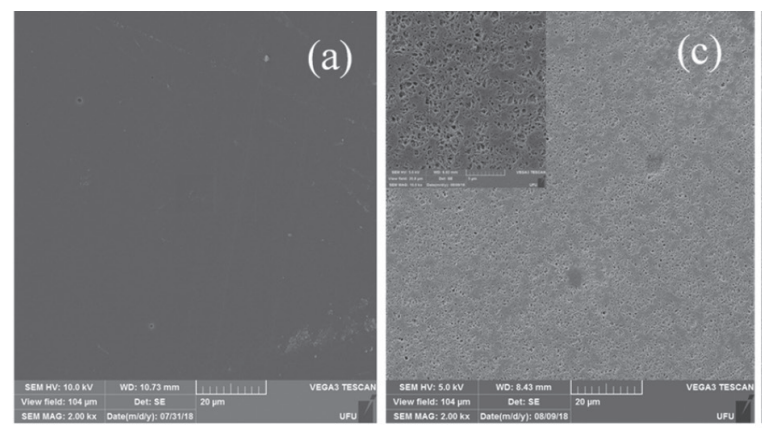

(b)
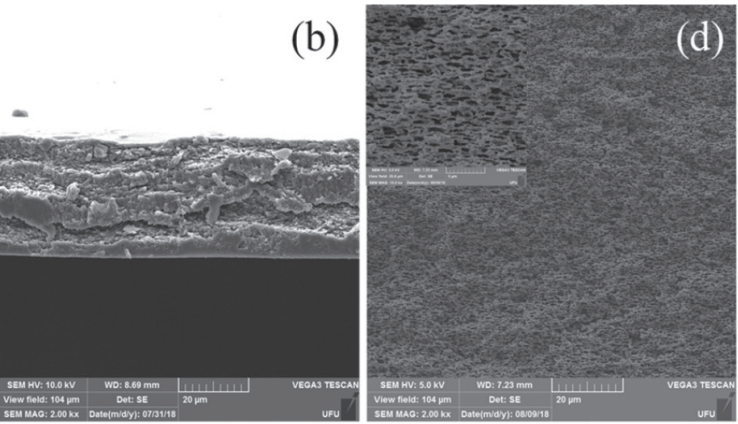
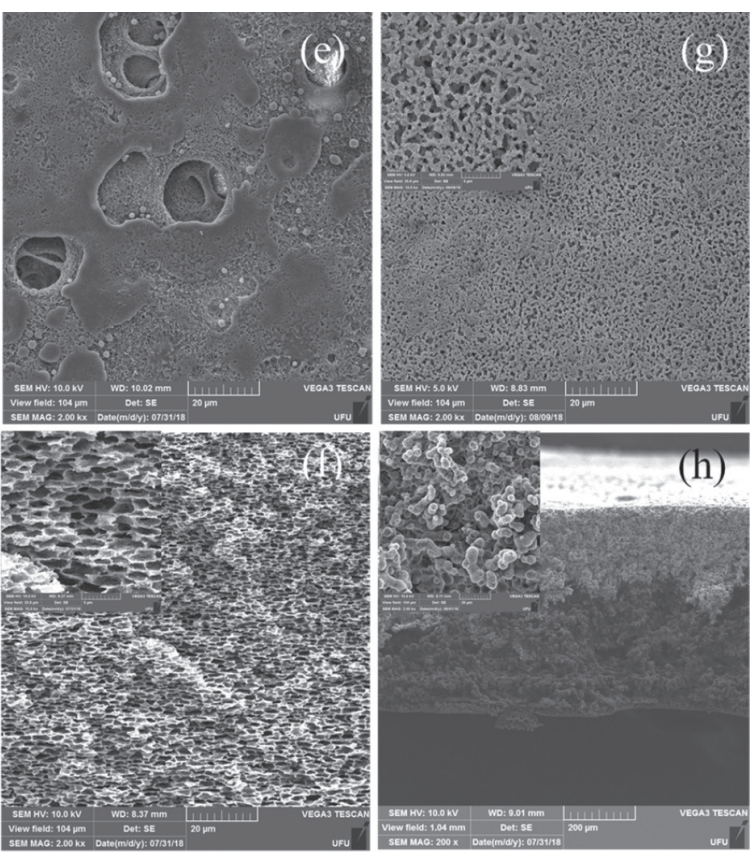

Figura 3. Imagens por MEV da superfície e seção transversal das membranas referência: AC-SM (a, b); AC-AM10 (c, d); AC-AM15 (e, f); $A C$-AM25 (g, $h$ )
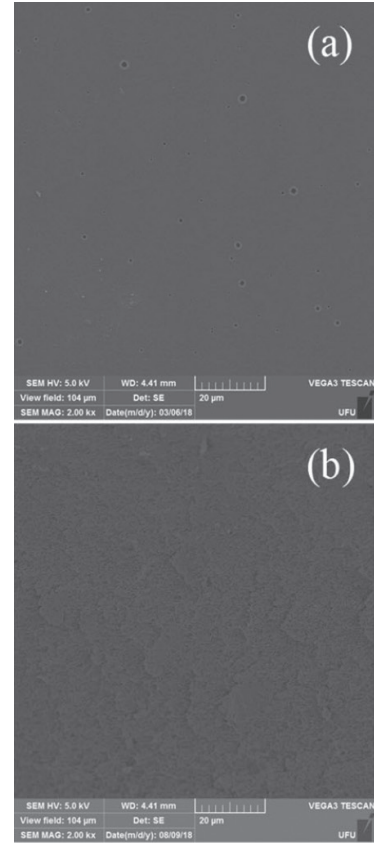
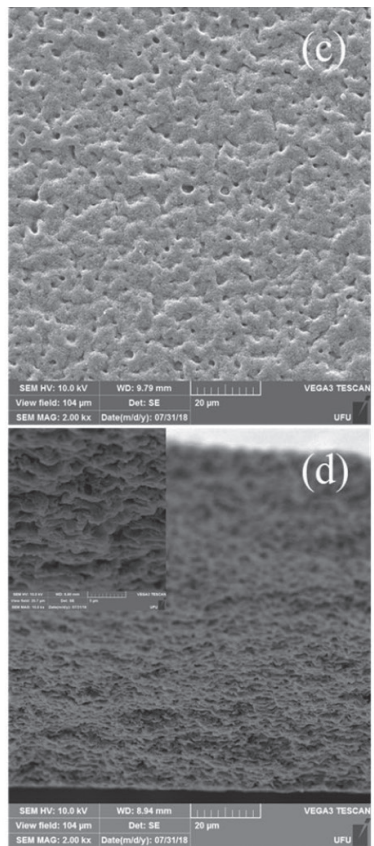
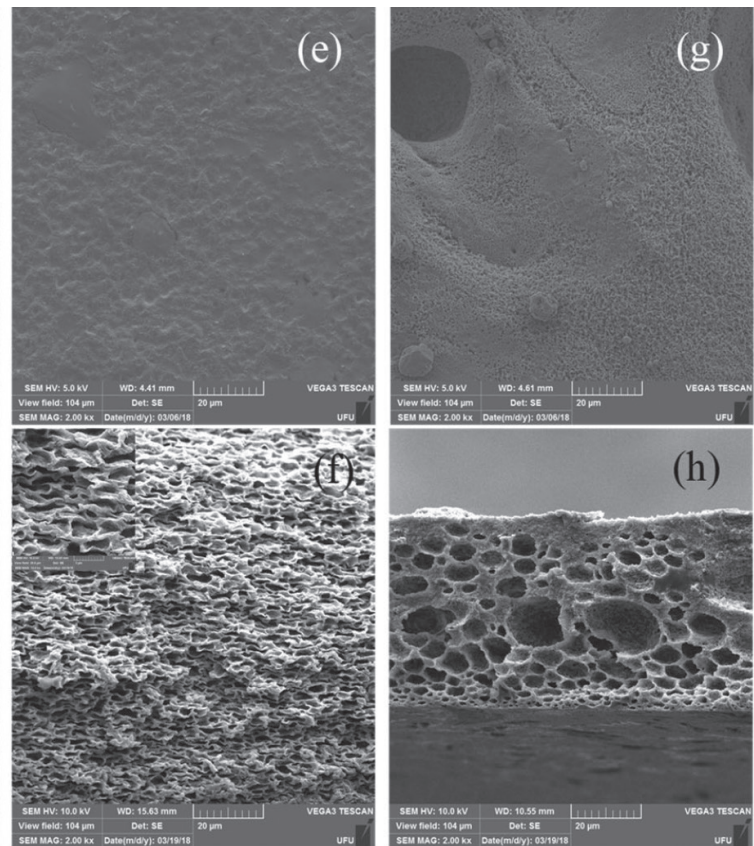

Figura 4. Imagens por MEV da superfície e seção transversal das membranas de AC-SM/IBF (a,b);AC-AM10/IBF (c, d); $A C$-AM15/IBF (e, f); $A C$-AM25/IBF ( $g$, $h$ )

No entanto, a adição de NS induz a uma separação de fases que culmina na formação da membrana pela precipitação do polímero pelo contato com a fase NS, bem como pela evaporação do solvente da solução polimérica. Assim, além dos fatores como umidade, atmosfera saturada do solvente e temperatura que comumente alteram o processo de formação de membranas pelo método de evaporação de solvente, têm-se o acréscimo do fármaco. Esse, na solução polimérica, pode agir como aditivo, alterando sua viscosidade e modificando as taxas de evaporação tanto do solvente como do NS e, consequentemente, as morfologias das membranas produzidas nestas condições. ${ }^{23,24}$ Desta forma, as membranas AC-AM10/IBF e AC-AM15/IBF apresentaram morfologias próximas entre si, porém bem distintas das membranas referência (sem fármaco).

Cabe ressaltar que, IBF apresenta valores dos componentes de parâmetro de solubilidade $(\delta)$ mais próximos do solvente acetona do que do polímero $\mathrm{AC}$, conforme pode ser visto nos dados apresentados na Tabela 1. Sendo assim, pode-se deduzir a partir da Equação 1, que a miscibilidade do fármaco no solvente deve ser mais favorável do que a miscibilidade deste no polímero. ${ }^{18,25}$

$$
\Delta \mathrm{H}_{\mathrm{m}}=\varphi_{1} \varphi_{2}\left[\left(\delta_{\mathrm{d} 1}-\delta_{\mathrm{d} 2}\right)^{2}+\left(\delta_{\mathrm{p} 1}-\delta \mathrm{p}_{2}\right)^{2}+\left(\delta_{\mathrm{h} 1}-\delta_{\mathrm{h} 2}\right)^{2}\right]
$$

Subscritos 1 e 2 se referem ao IBF e AC (ou acetona), respectivamente; $\varphi_{i}$ são frações volumétricas; $\delta_{\mathrm{d}}, \delta_{\mathrm{p}}$ e $\delta_{\mathrm{h}}$ são os componentes de dispersão, polar e de ligação de hidrogênio, respectivamente, dos parâmetros de solubilidade de Hildebrand e $\Delta \mathrm{H}_{\mathrm{m}}$ é a entalpia da mistura. ${ }^{18}$

Essa maior miscibilidade do IBF em acetona do que no polímero diminui a pressão de vapor do solvente utilizado, reduzindo a taxa de evaporação desse e modificando o processo de formação da estrutura 
Tabela 1. Parâmetros de Solubilidade para IBF, acetona e AC e Entalpias das Misturas calculadas para as frações utilizadas neste trabalho

\begin{tabular}{|c|c|c|c|c|c|c|c|c|}
\hline & $\delta_{\mathrm{d}}(\mathrm{MPa})^{0,5}$ & $\delta_{\mathrm{p}}(\mathrm{MPa})^{0,5}$ & $\delta_{\mathrm{h}}(\mathrm{MPa})^{0,5}$ & $\delta_{\mathrm{T}}(\mathrm{MPa})^{0,5}$ & & $\varphi_{1}$ & $\varphi_{2}$ & $\Delta \mathrm{H}_{\mathrm{m}}\left(\mathrm{J} \mathrm{m}^{-3}\right)$ \\
\hline IBF & 12,90 & 6,30 & 12,30 & 18,90 & IBF & 0,01 & -- & 0,52 \\
\hline Acetona & 15,55 & 10,43 & 6,95 & 19,97 & Acetona & -- & 0,99 & \\
\hline \multirow[t]{2}{*}{$\mathrm{AC}$} & 18,60 & 12,70 & 11,00 & 25,06 & IBF & 0,10 & -- & 6,76 \\
\hline & & & & & $\mathrm{AC}$ & -- & 0,90 & \\
\hline
\end{tabular}

Valores dos parâmetros de solubilidade retirados de Ma et al. ${ }^{18} \mathrm{e}$ Kitak et $a l .{ }^{26}$

porosa das membranas pelo método de evaporação de solvente. Com uma evaporação mais lenta do solvente, a formação da fase mais concentrada de polímero e posterior precipitação é retardada, permitindo que se formem poros de tamanhos e distribuições mais uniformes, conforme morfologias observadas nas Figuras 4c-h. O aumento da fração de NS nas soluções poliméricas acompanhou o aumento no tamanho dos poros (cerca de $2 \mu \mathrm{m}$ para AC-AM10/IBF e de 4 a $6 \mu \mathrm{m}$ para AC-AM15/IBF), chegando a formar macroespaços vazios como os da membrana AC-AM25/IBF de dimensões de cerca de $20 \mu \mathrm{m}$.

Outra consequência associada à proximidade entre os valores dos parâmetros de solubilidade do IBF e acetona é que, esse último sendo o solvente utilizado para o AC, há premissa para a afirmação de que o IBF tenha boa interação com o polímero, possibilitando sua dispersão molecular na matriz dependendo da porcentagem de fármaco adicionada.
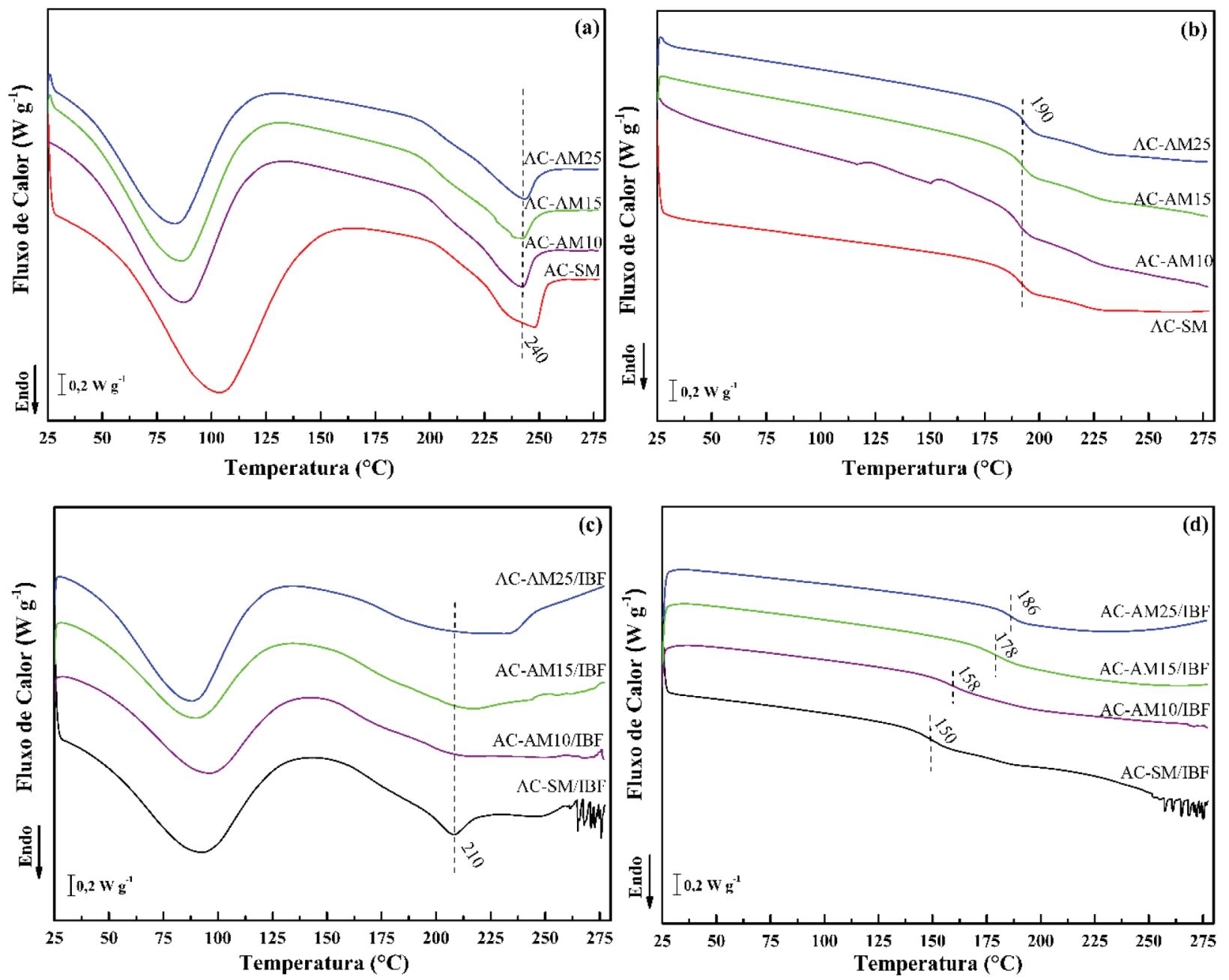

(c)

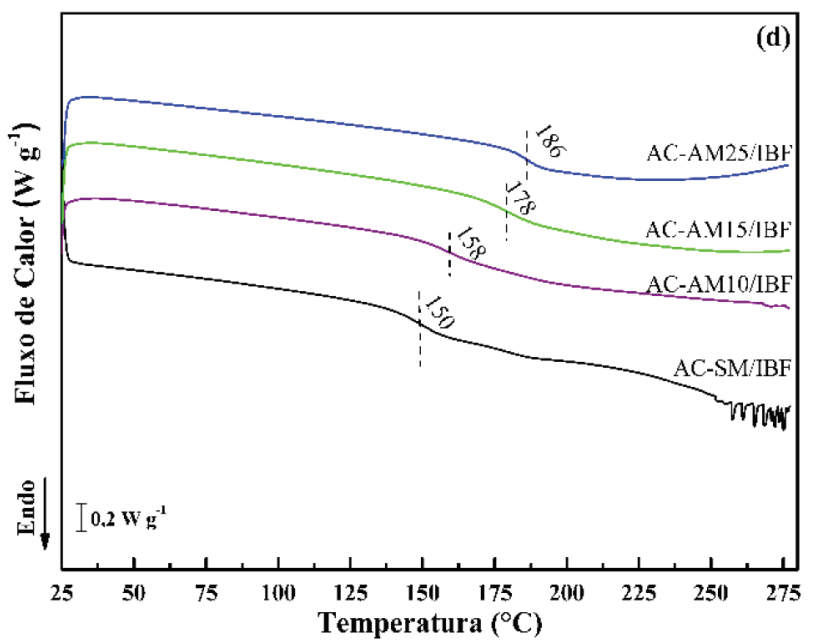

Figura 5. Curvas DSC das membranas referência e contendo IBF (a e c, respectivamente) para primeira e (b e d) segunda varredura. Taxa de aquecimento de $10{ }^{\circ} \mathrm{C} \mathrm{min}^{-1}$ e fluxo de $\mathrm{N}_{2}$ a $50 \mathrm{~cm}^{3} \mathrm{~min}^{-1}$ 
Tabela 2. Temperaturas $\left({ }^{\circ} \mathrm{C}\right)$ e Entalpias $\left(\mathrm{J} \mathrm{g}^{-1}\right)$ associadas aos eventos endotérmicos presentes nas curvas de DSC de primeira varredura e $\mathrm{T}_{\mathrm{g}}\left({ }^{\circ} \mathrm{C}\right)$ para a segunda varredura das membranas referência e carregadas com IBF. Taxa de aquecimento de $10{ }^{\circ} \mathrm{C} \mathrm{min}^{-1}$ e fluxo de $\mathrm{N}_{2}$ a $50 \mathrm{~cm}^{3} \mathrm{~min}^{-1}$

\begin{tabular}{|c|c|c|c|c|c|}
\hline Amostras & $\Delta \mathrm{H}_{\mathrm{dH}_{2} \mathrm{O}}\left(\mathrm{J} \mathrm{g}^{-1}\right)$ & $\mathrm{T}_{\mathrm{d}}\left({ }^{\circ} \mathrm{C}\right)$ & $\Delta \mathrm{H}_{\text {fusão }}\left(\mathrm{J} \mathrm{g}^{-1}\right)$ & $\mathrm{T}_{\mathrm{f}}\left({ }^{\circ} \mathrm{C}\right)$ & $\mathrm{T}_{\mathrm{g}}\left({ }^{\circ} \mathrm{C}\right)$ \\
\hline AC-SM & 107,10 & 103,48 & 18,27 & 246,83 & 191,46 \\
\hline AC-AM10 & 71,38 & 86,99 & 13,78 & 240,80 & 190,57 \\
\hline AC-AM15 & 71,05 & 85,78 & 13,40 & 238,15 & 191,32 \\
\hline AC-AM25 & 62,38 & 83,16 & 13,48 & 242,24 & 192,64 \\
\hline AC-SM/IBF & 62,76 & 91,91 & 11,34 & 207,52 & 150,40 \\
\hline AC-AM10/IBF & 57,83 & 95,22 & 21,28 & 207,51 & 158,37 \\
\hline AC-AM15/IBF & 53,06 & 89,01 & 18,36 & 209,52 & 178,63 \\
\hline AC-AM25/IBF & 73,38 & 87,85 & 25,73 & 211,51 & 186,69 \\
\hline
\end{tabular}

térmicas encontradas para as membranas. Todas as propriedades investigadas: temperatura de fusão, $\mathrm{T}_{\mathrm{f}}$; temperatura de transição vítrea, $\mathrm{T}_{\mathrm{g}}$; temperatura de dessorção de água, $\mathrm{T}_{\mathrm{d}}$; Entalpia de dessorção de água, $\Delta \mathrm{H}_{\mathrm{dH}_{2} \mathrm{O}}$; e Entalpia de fusão, $\Delta \mathrm{H}_{\text {fusão }}$; são modificadas em maior ou menor grau por propriedades do sistema tais como porcentagem de agente porogênico, porcentagem de fármaco e interações com o sistema polímero-solvente-fármaco.

Os dados da Tabela 2 mostram que a mudança da morfologia das membranas referência (sem fármaco) levam a alteração de $\Delta \mathrm{H}_{\mathrm{dH}_{2} \mathrm{O}} \mathrm{e}$ da $\mathrm{T}_{\mathrm{d}}$, com a diminuição destes valores. A diminuição dos valores indica que o processo de dessorção é facilitado. Isso pode estar relacionado à maior porosidade das membranas que favorece a dispersão da água na membrana e também sua dessorção, uma vez que esta pode estar superficialmente ligada. A diminuição de $\Delta \mathrm{H}_{\text {fusão }}$ e da $\mathrm{T}_{\mathrm{f}}$ do polímero pode estar associada a uma dificultação da formação dos domínios cristalinos na presença do não solvente, porém, essa perturbação não é extensiva. Para as membranas sem os fármacos, pouca mudança nas $\mathrm{T}_{\mathrm{g}}$ são observadas de uma membrana para outra, indicando que a presença da água não altera significativamente a mobilidade das cadeias poliméricas, atuando assim como agente porogênico e não como agente plastificante.

Para as membranas carregadas com IBF, o primeiro aspecto marcante é a ausência de uma endoterma de fusão do IBF que, de acordo com a literatura, ocorre por volta de $76^{\circ} \mathrm{C}$, não sendo registrada nem na primeira ou na segunda varredura das curvas térmicas. ${ }^{29}$ Esse aspecto indica que o fármaco foi disperso molecularmente no filme polimérico sólido, constituindo a formação de uma boa mistura entre polímero e fármaco.

Outro fator que corrobora a incorporação do fármaco e o estabelecimento de interações deste com a matriz, são os valores de $\mathrm{T}_{\mathrm{g}}$ que, para as membranas com o fármaco, apresentam uma diferença entre 10 a $40{ }^{\circ} \mathrm{C}$ em relação as membranas referência. Neste caso, evidencia também a atuação do fármaco como agente plastificante, cujo efeito é menos pronunciado quando a membrana é produzida com menor quantidade de agente porogênico.

Devido ao efeito plastificante, a presença do fármaco também diminui $\Delta \mathrm{H}_{\text {fusão }}$ e $\mathrm{T}_{\mathrm{f}}$ do polímero. ${ }^{30}$ Portanto, pode-se afirmar que as maiores reduções na $\mathrm{T}_{\mathrm{g}}$ foram para as membranas $\mathrm{AC}-\mathrm{SM} / \mathrm{IBF}$, AC-AM10/IBF e AC-AM15/IBF, nessa ordem (de 191,46 para $150,40{ }^{\circ} \mathrm{C}$; 190,57 para $158,37{ }^{\circ} \mathrm{C}$ e 191,32 para $178,63{ }^{\circ} \mathrm{C}$, respectivamente), em relação às membranas referência e indicam que a interação polímero-fármaco (P-F) é tanto maior quanto maior a redução no valor da $T_{\mathrm{g}}$.

Essa afirmação pode ser avaliada pelos resultados dos ensaios de liberação do fármaco, uma vez que esses dependem (entre outros fatores) da interação estabelecida entre a matriz polimérica e o fármaco no meio de teste. A consequência direta disso é que se espera para a membrana AC-AM25/IBF, uma liberação mais rápida do fármaco da matriz, uma vez que sua $\mathrm{T}_{\mathrm{g}}$ é pouco menor que a da membrana referência AC-AM25 $\left(186,69^{\circ} \mathrm{C}\right.$ e $192,64{ }^{\circ} \mathrm{C}$, respectivamente), ou seja, esta deve possuir interação P-F menos intensa.

\section{Ensaios de liberação in vitro do fármaco IBF}

Os perfis de liberação do IBF das membranas de AC foram obtidos a partir da detecção e quantificação deste por SWV, seguindo um procedimento análogo ao descrito por Fonseca et al. ${ }^{3}$ As curvas de liberação são apresentadas na Figura 6.

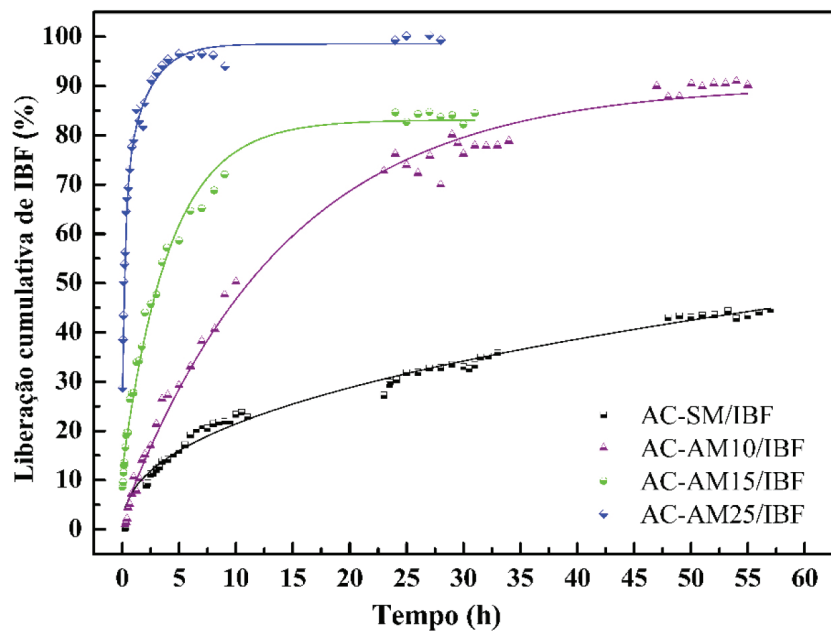

Figura 6. Ensaios de liberação cumulativa do fármaco $I B F(\%)$ das membranas AC-SM/IBF (-); AC-AM10/IBF (-); AC-AM15/IBF (-) e AC-AM25/IBF (-), quantificado por $S W V$, em função do tempo (h)

O perfil de liberação do IBF da membrana simétrica AC-SM/IBF exibe a menor porcentagem de fármaco liberado durante os testes para as membranas contendo IBF, alcançando um valor de aproximadamente $45 \%$ de liberação após cinquenta e sete horas de ensaio. Esse resultado está associado ao fato de que esta membrana possui a estrutura morfológica mais densa dentre as preparadas, o que dificulta a entrada do fluido de simulação intestinal (FSI), tampão fosfato $0,1 \mathrm{~mol} \mathrm{~L}^{-1}, \mathrm{pH} 7,4$; e, consequentemente, a difusão do fármaco para fora da membrana. Além disso, deve-se considerar que os resultados das análises térmicas, principalmente o menor valor de $\mathrm{T}_{\mathrm{g}}$, indicaram uma interação mais intensa P-F para esta membrana, justificando o perfil de liberação obtido.

AC-AM10/IBF apresentou um perfil de liberação contínua e lenta do fármaco, porém, com uma porcentagem de liberação mais elevada. 
Tabela 3. Parâmetros cinéticos calculados pelos modelos cinéticos e estatísticos avaliados

\begin{tabular}{|c|c|c|c|c|c|c|c|c|c|}
\hline \multirow[b]{2}{*}{ Amostras } & \multicolumn{8}{|c|}{ Modelos } & \multirow{2}{*}{ 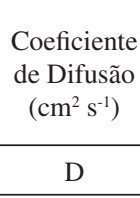 } \\
\hline & \multicolumn{2}{|c|}{$\begin{array}{c}\text { Higuchi } \\
\mathrm{M}_{\mathrm{t}} / \mathrm{M}_{\infty}=\mathrm{k}_{\mathrm{H}} \mathrm{t}^{1 / 2}\end{array}$} & \multicolumn{3}{|c|}{$\begin{array}{c}\text { Korsmeyer-Peppas } \\
\mathrm{M}_{\mathrm{t}} / \mathrm{M}_{\infty}=\mathrm{kt}^{\mathrm{n}}\end{array}$} & \multicolumn{3}{|c|}{$\begin{array}{c}\text { Weibull } \\
\mathrm{M}_{\mathrm{t}} / \mathrm{M}_{\infty}=1-\mathrm{e}^{\left(-\mathrm{at}^{\mathrm{b}}\right)}\end{array}$} & \\
\hline AC-SM/IBF & 0,967 & 0,062 & 0,989 & 0,081 & 0,425 & 0,985 & 0,077 & 0,501 & $1,51 \times 10^{-11}$ \\
\hline AC-AM10/IBF & 0,901 & 0,134 & 0,974 & 0,142 & 0,482 & 0,996 & 0,099 & 0,712 & $9,95 \times 10^{-11}$ \\
\hline AC-AM25/IBF & 0,927 & 0,901 & 0,911 & 0,590 & 0,213 & 0,992 & 1,573 & 0,427 & $3,72 \times 10^{-08}$ \\
\hline
\end{tabular}

Para essa, foram necessárias vinte e oito horas para se atingir cerca de $80 \%$ de IBF liberado e cinquenta e seis horas para liberar praticamente $100 \%$ do fármaco. Essa quantidade de fármaco liberado e o perfil de liberação obtido podem ser explicados em parte pela morfologia desta membrana de poros menores (diâmetro de cerca de $2 \mu \mathrm{m}$ ), achatados, e mais ordenados (Figura 4c e 4d), que tornam o acesso do FSI ao fármaco disperso na matriz polimérica mais facilitado do que para AC-SM/IBF, como também pela menor interação P-F formada em relação a este último, prevista pela $\mathrm{T}_{\mathrm{g}}$.

Dessa forma, considerando-se o tempo para obtenção das concentrações séricas máximas e tempo de meia-vida do IBF em formulações orais convencionais, destaca-se que os perfis de liberação obtidos para AC-SM/IBF e AC-AM10/IBF os qualificam para o tratamento de enfermidades crônicas como a artrite reumatoide ou osteoartrite, já que esses tratamentos necessitam de um fornecimento contínuo por longos períodos de tempo, maximizando a eficiência de cada dose e, assim, reduzindo o número de doses subsequentes requeridas.

Para AC-AM15/IBF, a porcentagem de fármaco liberado ao final de trinta e duas horas de ensaio é de cerca de $85 \%$, porém $75 \%$ do IBF são liberados nas primeiras oito horas de ensaio. Essa diminuição no tempo requerido para liberação de tais porcentagens de fármaco está relacionada ao aumento no tamanho dos poros em relação aos de AC-AM10/IBF, evidenciados pela seção transversal vista na Figura 4f, que favorece a entrada do FSI e, consequentemente, a difusão do fármaco da matriz, além da diminuição nas forças de interação P-F, indicadas pelo valor da $\mathrm{T}_{\mathrm{g}}$ deste material.

Em AC-AM25/IBF, uma liberação inicial de cerca de $25 \%$ do fármaco é observada logo nos minutos iniciais de teste e está associado à liberação de parte do fármaco que se encontra distribuído mais superficialmente que, de acordo com as previsões feitas durante a discussão dos resultados das curvas de DSC de que a interação P-F é menor para esse material (evidenciado pelo valor da $\mathrm{T}_{\mathrm{g}}$ próximo ao da membrana referência) em comparação com as demais membranas, ao entrar em contato com a solução FSI resulta nesta liberação inicial rápida. À parte dessa porcentagem de liberação inicial, esta membrana consegue atingir cerca de $95 \%$ de liberação do fármaco nas quatro primeiras horas de ensaio. Isso se deve à influência do IBF na modificação morfológica destas membranas que para AC-AM25/IBF, apesar de possuir poros de tamanhos que variam entre $2 \mathrm{e}$ $10 \mu \mathrm{m}$ e macroespaços vazios de cerca de $20 \mu \mathrm{m}$, essas ainda apresentam uma estrutura porosa mais uniforme do que AC-AM25.

Para essas, também considerando-se o tempo para obtenção das concentrações séricas máximas e tempo de meia-vida do IBF em formulações orais convencionais, têm-se que os perfis de liberação obtidos para AC-AM15/IBF e AC-AM25/IBF, por sua vez, os tornam elegíveis para o tratamento de enfermidades agudas como tratamento de dor leve a moderada e redução de febre, por exemplo, diminuindo a quantidade de doses necessárias, uma vez que o fármaco é liberado gradativamente em intervalos que podem atingir oito horas.
Os resultados mostram que as membranas com água em sua formulação apresentam menor densificação e, portanto, facilidade para a migração do fármaco para o FSI. Maior retenção é observada para a membrana simétrica densa, como esperado.

\section{Aplicação de Modelos Cinéticos às Curvas de Liberação do IBF}

Os perfis de liberação foram avaliados a partir dos modelos cinéticos de Higuchi, Korsmeyer-Peppas e o modelo estatístico de Weibull. ${ }^{15}$ Os resultados são apresentados na Tabela 3.

Os resultados apresentados na Tabela 3 mostram que o mecanismo de transporte das membranas é melhor modelado pelo modelo de Korsmeyer/Peppas que pelo modelo de Higuchi. O modelo de Higuchi vem sendo aplicado a sistemas matriciais homogêneos que seguem a lei de Fick. O tratamento $n=0,5$ é o limite superior para propor a cinética como um processo de solução/difusão através da matriz. ${ }^{15}$ Considerando a aplicação do modelo de Korsmeyer/Peppas, verificouse que o ajuste dos dados nesse modelo confirma o mecanismo de solução/difusão uma vez que $n$ é próximo de 0,5 para os sistemas com 0 e $10 \%$ de agente porogênico. Para os sistemas com 15 e $25 \%$ de agente porogênico, o valor do parâmetro $n$, que indica o mecanismo de transporte, fica entre 0,20 e 0,32 , o que reforça o mecanismo por solução/difusão, apesar da presença dos poros. Esses valores de $n$ menores que 0,5 podem indicar um processo pseudo-Fickiano, em que as curvas de difusão são muito semelhantes a um processo Fickiano, entretanto, atinge o equilíbrio de liberação mais rapidamente. ${ }^{31}$

Uma vez que a constante $k$, do modelo Korsmeyer/Peppas, incorpora informações estruturais, geométricas e de controle cinético das matrizes, o aumento dessa constante acompanhando o aumento da razão de NS adicionado, indica que a cinética de liberação do fármaco é favorecida pelas modificações morfológicas promovidas, neste trabalho. ${ }^{15}$

Uma análise mais aprofundada mostra que os modelos de Higuchi e Korsmeyer/Peppas, apesar de aplicáveis, apresentam valores de coeficiente de determinação, $\mathrm{R}^{2}$, que indicam que entre 3 e $10 \%$ dos dados dos perfis de liberação não se ajustam aos modelos testados. Essa deficiência no ajuste dos dados experimentais pode ser atribuída à inadequação do modelo e/ou a uma certa dispersão dos resultados experimentais.

Para melhor avaliar estes sistemas foi aplicado o modelo de Weibull. Essa é uma equação empírica aplicada aos processos de dissolução/liberação controlada. Quando aplicada a esses sistemas, a equação expressa a fração de fármaco acumulada na solução no tempo $t$ de acordo com a Equação 2:

$$
M_{t} / M_{\infty}=1-e^{\left(-a t^{b}\right)}
$$

A utilização dessa expressão empírica sempre foi restrita devido à ausência de base cinética para seu uso e a natureza não física dos seus parâmetros. Porém, Papadopoulou et al. ${ }^{32}$ foram capazes de obter uma relação linear entre o expoente $n$ da expressão de Korsmeyer/ 
Peppas e o expoente $b$ do tempo $t$ da expressão de Weibull. Para os autores, $b$ também é considerado um indicador do mecanismo de transporte através da matriz em que, quando $b \leq 0,75$, pode ser relacionado a $n \leq 0,5$ da expressão de Korsmeyer/Peppas, indicando que é o mecanismo de difusão Fickiano que regula a liberação a partir das membranas testadas e corroborando a afirmação oferecida pelo modelo de Korsmeyer/Peppas.

Os resultados dos Coeficientes de Difusão (D) para as membranas produzidas neste trabalho, descritos na Tabela 3 , traduzem os perfis de liberação obtidos ao evidenciar o aumento na velocidade de difusão do fármaco para fora da matriz polimérica conforme foram realizadas as modificações morfológicas testadas.

\section{CONCLUSÃO}

Este trabalho mostrou que a morfologia das membranas AC pode ser eficientemente modificada pela adição de água como agente de formação de poros, garantindo a formação de matrizes assimétricas menos densas do que as membranas de AC simétricas. As caracterizações empregadas permitem afirmar que a quantidade de fármaco adicionada às membranas garantiu sua dispersão na matriz polimérica. $\mathrm{O}$ IBF incorporado às membranas modifica a matriz, exercendo um efeito plastificante de destacada importância na liberação do fármaco no fluido de simulação intestinal. O processo de liberação do IBF ocorre predominantemente por difusão/solução na matriz polimérica. Os resultados mostraram que a interação do fármaco com o polímero e a morfologia da membrana são importantes fatores no controle cinético do processo de liberação do fármaco, que culminaram em mudanças expressivas do tempo de liberação e na quantidade de fármaco retido, tornando as membranas candidatas viáveis para aplicação tanto no tratamento de doenças crônicas, como a artrite reumatoide, quanto no tratamento de enfermidades agudas, como antitérmico ou analgésico.

\section{AGRADECIMENTOS}

Os autores agradecem à CAPES (No 1687830) pelas bolsas de Mestrado e Doutorado concedidas, FAPEMIG (No APQ-02905-15 e APQ-02078-15), PROPP/UFU e FINEP pelo apoio financeiro e ao Laboratório Multiusuários do Instituto de Química da Universidade Federal de Uberlândia pela realização dos ensaios de DSC e MEV.

\section{REFERÊNCIAS}

1. Ramasamy, T.; Ruttala, H. B.; Gupta, B.; Poudel, B. K.; Choi, H. G.; Yong, C. S.; Kim, J. O.; J. Control. Release 2017, 258, 226.

2. Qiu, L. Y.; Bae, Y. H.; Pharm. Res. 2006, 23, 1.

3. Fonseca, W. T.; Santos, R. F.; Alves, J. N.; Ribeiro, S. D.; Takeuchi, R.
M.; Santos, A. L.; Assunção, R. M. N.; Filho, G. R.; Muñoz, R. A. A.; Electroanalysis 2015, 27, 1847.

4. Pelipenko, J.; Kocbek, P.; Kristl, J.; Int. J. Pharm. 2015, 484, 57.

5. Yu, D. G.; Li, X. Y.; Wang, X.; Chian, W.; Liao, Y. Z.; Li, Y.; Cellulose 2013, 20, 379.

6. Nista, S. V. G.; D’Ávila, M. A.; Martinez, E. F.; Silva, A. S. F.; Mei, L. H. I.; J. Appl. Polym. Sci. 2013, 130, 2772.

7. Kesting, R. E.; J. Appl. Polym. Sci. 1973, 17, 1771.

8. Shojaie, S. S.; Krantz, W. B.; Greenberg, A. R.; J. Membr. Sci. 1994, 94, 281.

9. Pekny, M. R.; Greenberg, A. R.; Khare, V.; Zartman, J.; Krantz, W. B.; Todd, P.; J. Membr. Sci. 2002, 205, 11

10. Altinkaya, S. A.; Ozbas, B.; J. Membr. Sci. 2004, 230, 71.

11. Hite, M.; Federici, C.; Brunelle, A.; Turner, S.; US pat. 20060068009 A1 2006.

12. Bushra, R.; Aslam, N.; Oman Med. J. 2010, 25, 155.

13. Albert, K. S.; Gernaat, C. M.; Am. J. Med. 1984, 23, 40.

14. Rainsford, K. D.; Inflammopharmacology 2009, 17, 275.

15. Costa, P.; Lobo, J. M. S.; Eur. J. Pharm. Sci. 2001, 13, 123.

16. Švorc, L'.; Strežová, I.; Kianičková, K.; Stanković, D. M.; Otřísal, P.; Samphao, A.; J. Electroanal. Chem. 2018, 822, 144.

17. Sun, N.; Yan, X.; Wang, T.; RSC Adv. 2017, 7, 9500.

18. Ma, D.; McHugh, A. J.; J. Membr. Sci. 2007, 298, 156.

19. Singh, B.; Sharma, D. K.; Gupta, A.; J. Hazard. Mater. 2008, 154, 278.

20. Bao, C. Y.; Long, D. R.; Vergelati, C.; Carbohydr. Polym. 2015, 116, 95.

21. Ferreira Júnior, M. F.; Mundim, E. A. R.; Rodrigues Filho, G.; Meireles, C. S.; Cerqueira, D. A.; Assunção, R. M. N.; Marcolin, M.; Zeni, M.; Polym. Bull. 2011, 66, 377.

22. Kunst, B.; Sourirajan S.; Desalination 1970, 8, 139.

23. Mohsenpour, S.; Esmaeilzadeh, F.; Safekordi, A.; Tavakolmoghadam, M.; Rekabdar, F.; Hemmati, M.; J. Mol. Liq. 2016, 224, 776.

24. Nguyen, Q. T.; Alaoui, O. T.; Yang, H.; Mbareck, C.; J. Membr. Sci. 2010, 358, 13.

25. Tungprapa, S.; Jangchud, I.; Supaphol, P.; Polymer 2007, 48, 5030.

26. Kitak, T.; Dumičič, A.; Planinšek, O.; Šibanc, R.; Srčič, S.; Molecules 2015, 20, 21549.

27. Glasser, W. G.; Samaranayake, G.; Dumay, M.; Davé, V.; J. Polym. Sci., Part B: Polym. Phys. 1995, 33, 2045.

28. Melo, P. G.; Borges, M. F.; Ferreira, J. A.; Silva, M. V. B.; Ruggiero, R.; Int. J. Mol. Sci. 2018, 19, 1.

29. https://pubchem.ncbi.nlm.nih.gov/compound/3672, acessada em Setembro 2019.

30. Sperling, L. H.; Introduction to Physical Polymer Science, $4^{\text {th }}$ ed.; Wiley: New Jersey, 2006.

31. Crank, J.; The Mathematics of Difussion, $2^{\text {nd }}$ ed.; Oxford University Press: Bristol, 1975.

32. Papadopoulou, V.; Kosmidis, K.; Vlachou, M.; Macheras, P.; Int. J. Pharm. 2006, 309, 44. 